\title{
Protein Viability on Au Nanoparticles during an Electrospray and Electrostatic-Force-Directed Assembly Process
}

\author{
Shun Mao, ${ }^{1}$ Ganhua Lu, ${ }^{1}$ Kehan Yu, ${ }^{1}$ and Junhong Chen ${ }^{1,2}$ \\ ${ }^{1}$ Department of Mechanical Engineering, University of Wisconsin-Milwaukee, 3200 North Cramer Street, Milwaukee, WI 53211, USA \\ ${ }^{2}$ State Key Laboratory of Pollution Control and Resource Reuse, College of Environmental Science and Engineering, \\ The Institute for Advanced Materials and Nano Biomedicine, Tongji University, 1239 Siping Road, Shanghai 200092, China
}

Correspondence should be addressed to Junhong Chen, jhchen@uwm.edu

Received 13 October 2009; Accepted 7 December 2009

Academic Editor: Chao Lin

Copyright (c) 2010 Shun Mao et al. This is an open access article distributed under the Creative Commons Attribution License, which permits unrestricted use, distribution, and reproduction in any medium, provided the original work is properly cited.

\begin{abstract}
We study the protein viability on Au nanoparticles during an electrospray and electrostatic-force-directed assembly process, through which Au nanoparticle-antibody conjugates are assembled onto the surface of carbon nanotubes (CNTs) to fabricate carbon nanotube field-effect transistor (CNTFET) biosensors. Enzyme-linked immunosorbent assay (ELISA) and field-effect transistor (FET) measurements have been used to investigate the antibody activity after the nanoparticle assembly. Upon the introduction of matching antigens, the colored reaction from the ELISA and the change in the electrical characteristic of the CNTFET device confirm that the antibody activity is preserved during the assembly process.
\end{abstract}

\section{Introduction}

The protein viability has always been an issue in the biosensor design and fabrication, and is a key factor for the function of biosensing devices. In the past decade, carbon nanotubes (CNTs) with all atoms on the surface have been demonstrated for biomolecular sensing [1-3]; and in particular, field effect transistors (FETs) based on semiconducting CNTs have been used as biosensors [4-8]. The CNTFET devices are sensitive to variations in the surrounding environment because all the electrical current flows through the outermost layer of the CNT. The electrochemical detection of protein binding $[9,10]$ and DNA hybridization [11-14] using the CNTFET have been reported and changes in the device characteristics (e.g., drain current amplitude and threshold voltage) are mainly attributed to the charge transfer between proteins and CNTs. In these CNTFET devices [10-12], the CNT surface is often directly or indirectly (e.g., through $\mathrm{Au}$ nanoparticles) labeled with probe proteins, which function as binding sites for target proteins in the sensing process. Therefore, the viability of proteins during the assembly or labeling process is critical to the device functionality.
Here we investigate the viability of proteins on $\mathrm{Au}$ nanoparticles ( $\mathrm{Au}$ NPs) during an assembly process for the fabrication of CNTFET biosensors, in which Au NPs labeled with anti-horseradish peroxidase (anti-HRP) were assembled onto the external surface of CNTs by a simple method that combines electrospray with electrostatic force directed assembly (ESFDA) [15-17]. To study the viability of antibodies on Au NPs, we first used the enzyme-linked immunosorbent assay (ELISA) technique, which is mainly used in immunology to detect the presence of antibodies and antigens. For visualization of the antigen-antibody reaction, an enzyme and a related substrate are often introduced, which could offer a colored reaction that can be spectrophotometrically measured. In this study, the antigen-antibody binding reaction was indicated by the color change (diimine terminal oxidation product/yellow end product) from the $3,3^{\prime}, 5,5^{\prime}$-tetramethyl benzidine (TMB) substrate in the ELISA, in which horseradish peroxidase (HRP) functions as the antigen and enzyme. To further confirm the viability of antibodies, CNTFET devices containing the antibodyconjugated Au NPs were fabricated and FET measurements were obtained to detect the antigen-antibody binding events. The amplitude change of the drain current was observed 
when HRPs were introduced in the sensor. The above two independent approaches clearly show that the antibodies on $\mathrm{Au}$ NPs are still active after the electrospray and electrostatic force directed assembly process.

\section{Experimental}

2.1. Materials. Au nanoparticles (18 nm Colloidal Gold) labeled with anti-HRP were purchased from Jackson Immunoresearch and used without further purification. HRP from human serum were ordered from Sigma-Aldrich. A blocking agent bovine serum albumin (BSA), which was used to block the nonspecific binding of proteins with the sensing element, was purchased from Rockland. PBS ( $\mathrm{pH}$ $7.4, \times 1)$ (Fisher BioReagents) was used as the solvent for HRP and BSA. TMB and HCL were purchased from Bio-Rad Lab and VWR, respectively. All solutions were prepared with distilled and deionized water (DI water) supplied by Cellgro. Multiwalled CNTs (MWCNTs) with diameter of $20 \mathrm{~nm}$ and lengths of several microns were purchased from Alfa Aesar.

\subsection{Electrospray and Electrostatic Force Directed Assembly.} Figure 1 shows a schematic of Au nanoparticle-antibody conjugates aerosolization by an electrospray process and the subsequent assembly of the conjugates onto CNTs by ESFDA. A commercial electrospray aerosol generator (EAG, TSI Model 3480) was used to spray colloidal Au nanoparticleantibody conjugates. The colloidal conjugates applied with a high dc voltage were extracted through a capillary tube due to the capillary effect and the capillary inlet/outlet pressure difference. The conjugates ejected from the capillary were atomized to form charged fine droplets due to the electrohydrodynamic break-up. Charged Au NP-antibody conjugates were obtained after the solvent evaporation and subsequently assembled onto the surface of CNTs in an electric field. Since the electric field near the CNT was significantly enhanced due to their small diameters, the charged conjugates were attracted to the external surface of CNTs via electrostatic force. In our case, CNTs on a copper transmission electron microscopy (TEM) grid and $\mathrm{Au}$ electrodes were used. The assembly time was on the order of hours.

2.3. ELISA. 96-well polystyrene microplate (Sigma-Aldrich) was used as the reaction chamber in this procedure. At first, MWCNTs were dispersed on a copper TEM grid (400 mesh, Ted Pella, Inc.), and then Au nanoparticleantibody conjugates were assembled onto the CNTs by electrospray and ESFDA. Three samples with different antibody concentrations on the CNTs were prepared by adjusting the deposition time from one hour to three hours. The samples were placed in a microplate and incubated with $200 \mu \mathrm{L}$ BSA $(0.01 \mathrm{mg} / \mathrm{mL})$ for two hours, and then washed with the PBS buffer. After that, $100 \mu \mathrm{L}$ of HRP $(1.8 E-6 \mathrm{M}, 0.08 \mathrm{mg} / \mathrm{mL})$ were pipetted into the microplate for the protein binding for one hour, followed by wash and dry. Finally, $100 \mu \mathrm{L}$ of TMB substrates were pipetted into the microplate for the colored reaction; after 30 minutes, $100 \mu \mathrm{L}$ of $0.1 \mathrm{M} \mathrm{HCL}$ were pipetted to stop the reaction and to turn the solution into yellow which can be measured through the light absorption using a microplate reader.

2.4. CNTFET Fabrication and Measurement. Gold interdigitated electrodes with both finger width and interfinger spacing (source-drain separation) of about $1 \mu \mathrm{m}$ were fabricated using an e-beam lithography process (Raith 150 lithography tool, $30 \mathrm{kV}$ ) on a $\mathrm{Si}$ wafer with a top layer of thermally-formed $\mathrm{SiO}_{2}$ (thickness of $200 \mathrm{~nm}$ ). Firstly, MWCNTs were dispersed onto the electrodes and dried under room temperature. The device was then annealed in $\operatorname{argon}$ flow $(1 \mathrm{lpm})$ for one hour at $200^{\circ} \mathrm{C}$ to remove residue solvents and to improve the contact between CNTs and electrodes. After annealing, we found that the device was very robust. CNTs were immobilized between two metal electrodes and could not be washed away after several cycles of washing and drying, which has been confirmed by the SEM imaging. The device was then incubated with BSA for two hours and washed with the PBS buffer to reduce the possible nonspecific binding of HRP to CNTs and electrodes. After that, $20 \mu \mathrm{L}$ HRP was pipetted onto the device for protein binding for one hour, followed by wash and dry. FET measurements were carried out using a Keithley 2602 source meter by recording the drain current response $\left(V_{d}=0.01 \mathrm{~V}\right)$ when ramping the gate voltage $V_{g}$ from -3.0 to $3.0 \mathrm{~V}$ (with a step of $0.01 \mathrm{~V}$ ). A Hitachi S-4800 UHR FE-SEM was used for scanning electron microscopy (SEM) characterization of the biosensing device at an acceleration voltage of $30 \mathrm{kV}$.

\section{Results and Discussion}

For the ELISA technique (Figure 2(a)), three samples with increasing deposition time from one hour to three hours were prepared. All the samples were performed in ELISA with exactly the same procedure as described previously in Experimental. BSA was used to reduce the possible nonspecific binding of HRPs to the CNT, TEM grid and the well wall. In our case, the HRP functions as an enzyme, which converts the related substrate (TMB) to produce a yellow product. After adding the TMB substrate and HCL, the color of the solution turned yellow, which confirms the presence of HRPs on the sample. Since BSA was introduced to block the non-specific binding, HRPs could only bind with anti-HRP on the CNT. Therefore, the color change of the solution indicates that the antibody activity was preserved in the electrospray and ESFDA process.

Figure 2(b) shows the light absorption measurements for the three samples after the colored reaction. The absorption intensity increased with the deposition time. By adjusting the deposition time of electrospray and ESFDA, the concentration of Au NP-anti HRP conjugates on CNTs could be controlled. With more antibodies on CNTs, more HRPs can bind on the device, therefore leading to more converted yellow product. The absorption results further confirm that the anti-HRP activity is preserved and antigen-antibody binding events occurred in the ELISA. 


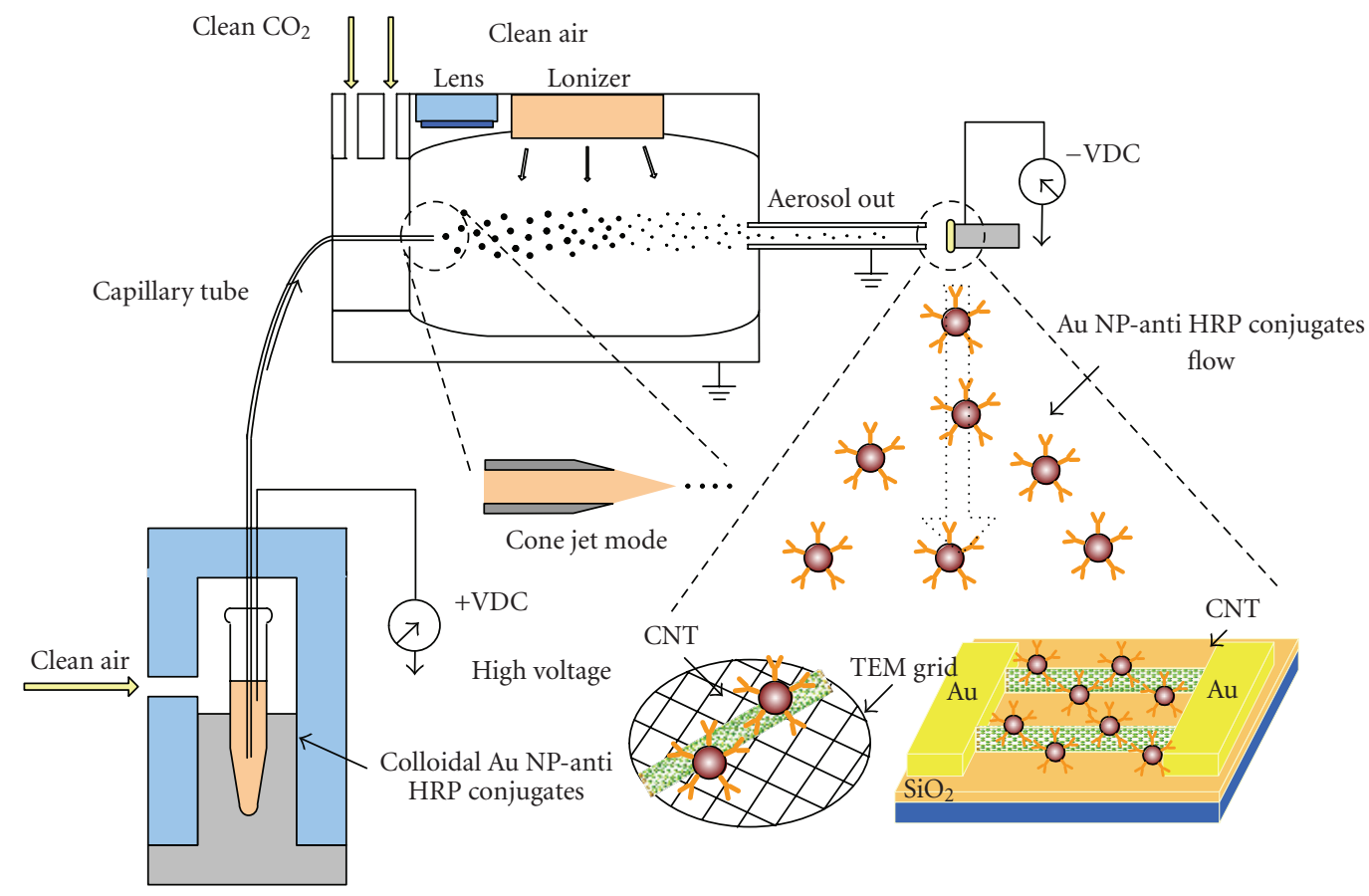

FIGURE 1: Schematic of Au nanoparticle-antibody conjugates aerosolization by an electrospray process and their subsequent assembly onto CNTs by ESFDA.

$\mathrm{Au}$ nanoparticle-antibody conjugates assembled on CNTs could be used in a CNTFET biosensor, from which the antibody activity could be studied by FET measurements. To confirm the presence of Au NPs on the surface of the CNT, SEM imaging was used to examine the CNTFET after the electrospray of Au NP-antibody conjugates. Figure 3(a) shows an SEM image of the $\mathrm{Au}$ interdigitated electrode [18]. In this configuration, Au electrodes are used as metal contacts for drain and source and the $\mathrm{SiO}_{2}$ layer is used as the insulating material, through which a gate bias is applied to the back silicon wafer during the FET measurement.

Figure 3(b) shows the SEM image of a single CNT coated with Au NP-anti HRP conjugates and spanning across an electrode gap. After being electrosprayed with Au NPantibody conjugates, many Au NPs (light dots) are uniformly distributed on the surface of the CNT, indicating the presence of Au NPs in the CNTFET. Based on the SEM images, we conclude that Au NPs have been successfully attached to the CNTs likely through noncovalent binding $[15,17]$. On the other hand, anti-HRPs are covalently linked to the surface of Au NPs and the bonding between antibody and Au NPs is relatively strong. Therefore, it is reasonable to assume that the antibodies are present in the CNTFET device after the assembly process. From the SEM image, we also found that the surface of the Au NPs was covered with a solvent layer, which is from the colloidal solution. It is known that the antibody is active for months when stored in buffer solution at $2-8^{\circ} \mathrm{C}$. In our case, the antibody on the Au NPs are covered with a solvent layer similar to the buffer solution, which prevents the metamorphose of the antibody. We assume that the antibodies are active in the sensor for probing antigens, which has been confirmed by the sensing results presented as follows.

Figure 4(a) shows the schematic illustration of the fabrication process of the CNTFET biosensor, in which the anti-HRP is anchored to the CNT surface through Au NPs and functions as the specific recognition group for HRP binding. FET measurements of the device were performed to study the antibody viability. Figure 4(b) shows the gate voltage dependence of the normalized drain current $I_{d}\left(I_{d}\right.$ is normalized by the drain current of the device at $V_{g}=$ $-3.0 \mathrm{~V}$ ) after the CNTFET was treated with Au NP-anti-HRP conjugates, BSA and HRP. During the FET measurement, a gate bias $V_{g}$ ramped from $-3.0 \mathrm{~V}$ to $3.0 \mathrm{~V}$ while a constant bias of $0.01 \mathrm{~V}$ was held between the drain and the source. Based on Figure 4(b), the device characteristics resemble those of typical p-type semiconductors, in which holes are the majority carriers and the conductivity of the CNT depends on the mobility and density of holes. No hysteresis was observed in all $V_{g}-I_{d}$ measurements. The type of device did not change after the CNTs and Au NP-antiHRP conjugates were further treated with BSA and HRP; however, the drain current $I_{d}$ decreased for both processes. The decrease in the drain current $I_{d}$ after the BSA treatment is possibly because BSA is negatively charged in the PBS buffer and negative charges could transfer to CNTs or electrons could transfer from the electron-rich group such as secondary amine $\mathrm{N}-\mathrm{H}$ in the BSA to CNTs, both of which could lead to the decrease in the density of holes in CNTs.

After the introduction of HRPs to the device, a significant decrease of $I_{d}$ (about $36 \%$ at $V_{g}=-3.0 \mathrm{~V}$ ) was observed, which is a direct evidence for antigen-antibody binding 


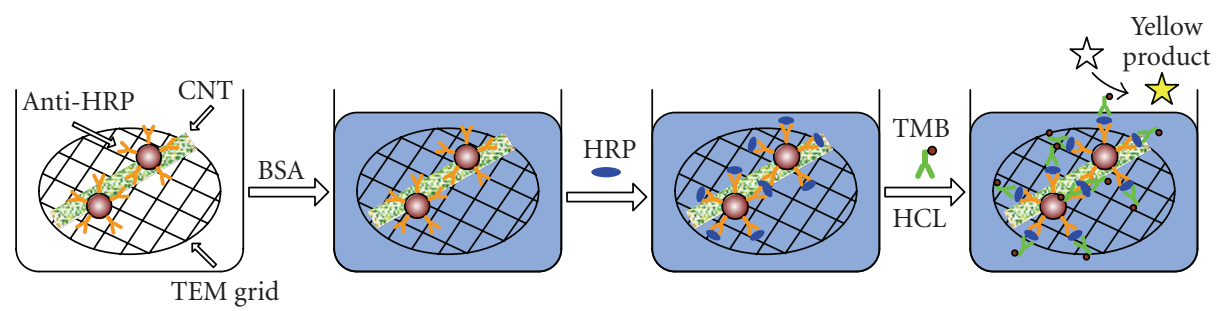

(a)

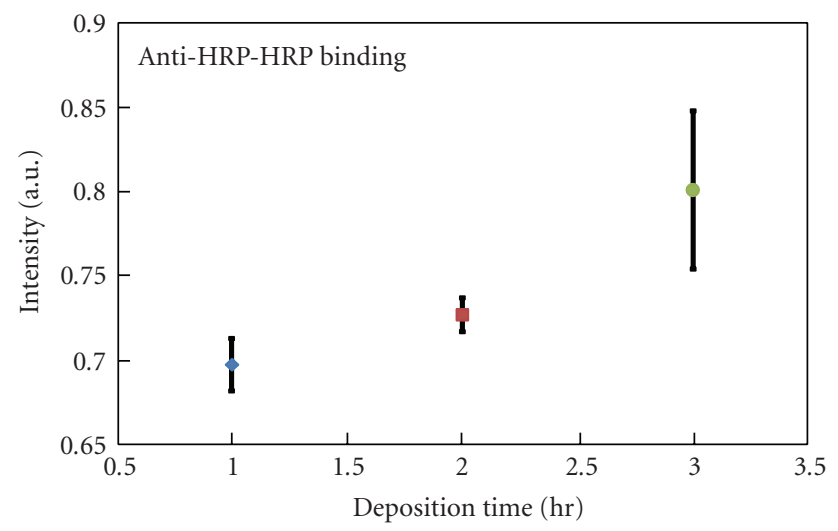

(b)

FIGURE 2: (a) Schematic illustration of ELISA. (b) Light absorption measurement from ELISA, in which HRP was added to three samples with different Au NP-anti HRP conjugates deposition time (1 hr, 2 hrs, and 3 hrs) and thus concentrations.

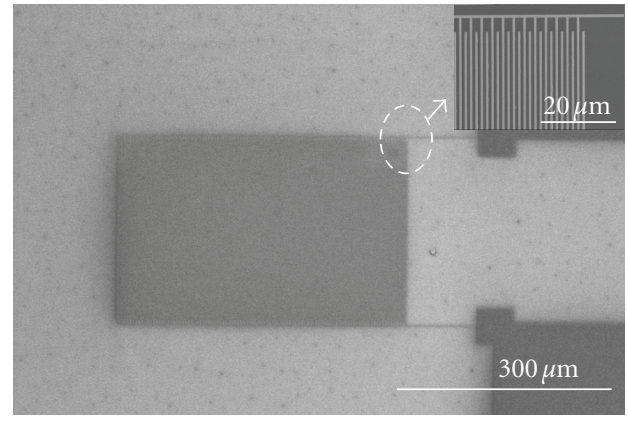

(a)

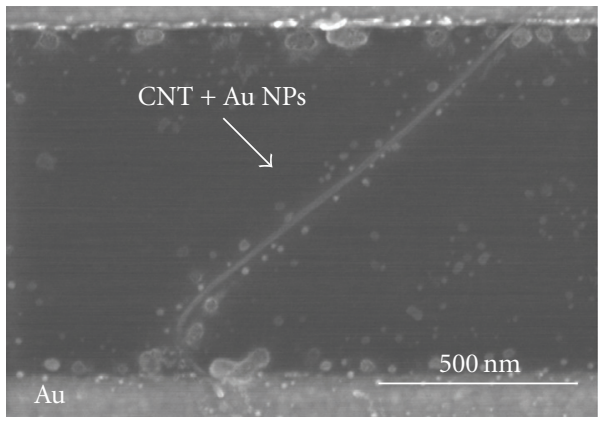

(b)

FIgURE 3: (a) SEM image of the Au interdigitated electrode. (b) SEM image of a single CNT coated with Au NP-anti HRP conjugates and spanning across an electrode gap.

events. The antibody-antigen interaction may have two effects on the CNT conductivity [5]. First, the antigen (HRP) triggers various scattering centers across the CNT as a result of binding events. Upon antigen-antibody binding, geometric deformations occur and increase the scattering centers across the tube; thereby reducing the mobility of holes in the CNT and thus leading to the decrease of drain current. As a possible second effect, the decrease of the drain current involves the charge tunneling from HRPs to CNTs. Charge tunneling from $\alpha$-amino acid groups in HRPs to the CNT through the solvent is possible in the buffer solution, which reduces the carrier density in CNTs and thus leads to the decrease in the drain current. The observed drain current decrease could be the results of both the scattering mechanism and the charge tunneling $[10,12]$. To study the solvent influence to the drain current, a control experiment was performed by introducing only the PBS buffer to the device and a very tiny response (5\% decrease in $I_{d}$ ) from the device was observed, indicating that the sensor response is mainly due to the binding of antigen to antibody. Figure 4(c) shows the drain-source voltage dependence of drain current $I_{d}$ for a CNTFET treated with HRP of concentrations ranging from $4.5 E-8 \mathrm{M}$ to $4.5 E-5 \mathrm{M}$. From the $I-V$ characteristics, we found that the drain current decreased monotonically with the increase of the HRP concentration, and this could only be explained by more antigens binding with antibodies, more scattering centers created, and more charge tunneling between CNTs and Au NPs. 


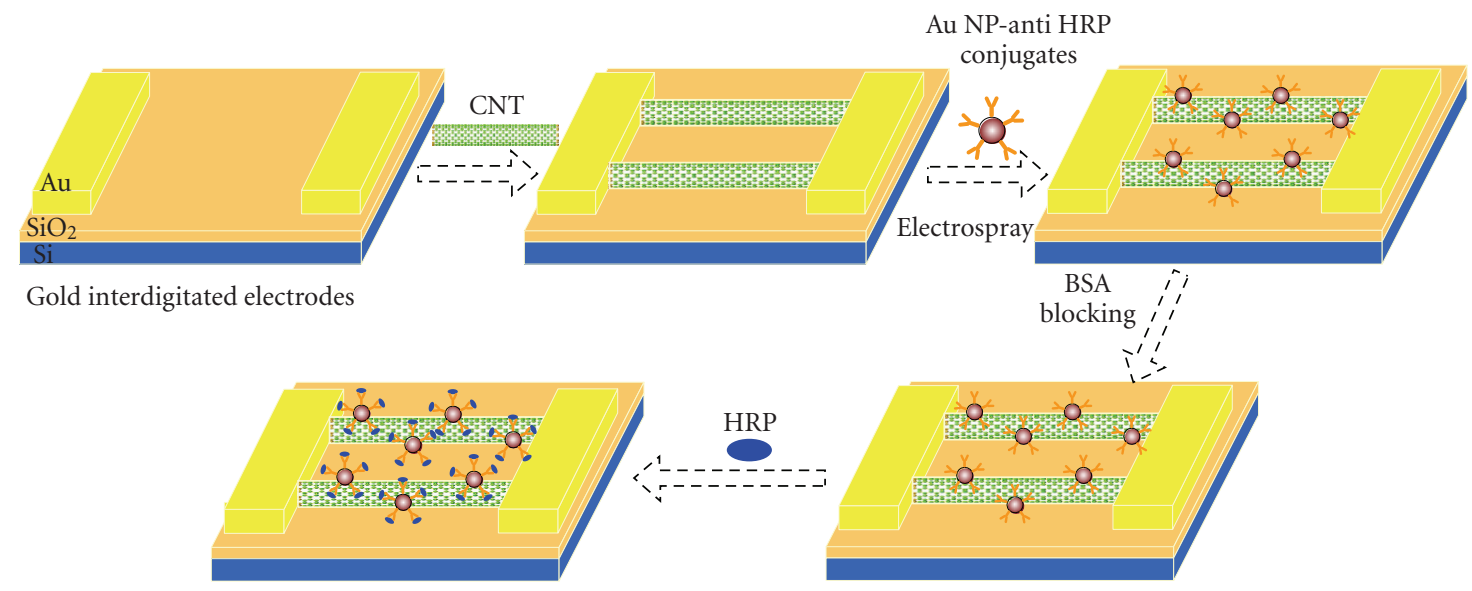

(a)

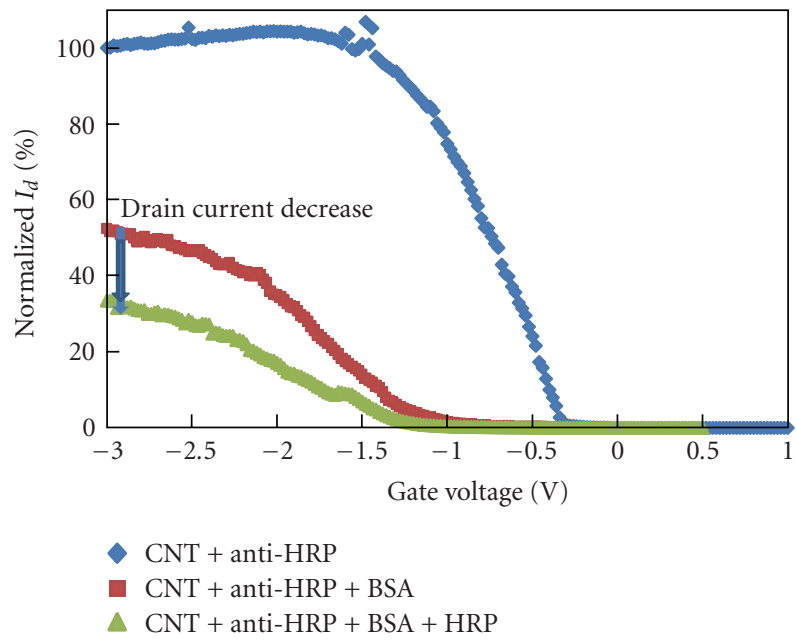

(b)

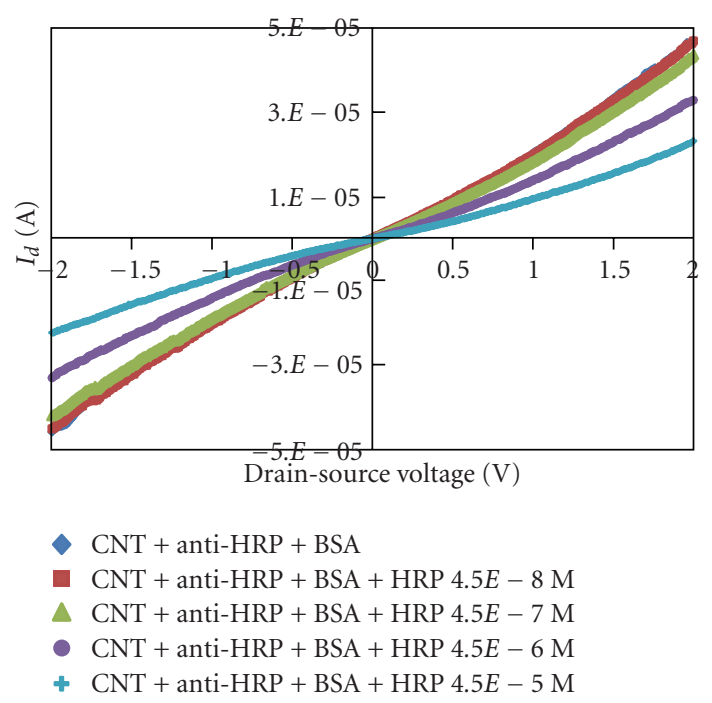

(c)

FIGURE 4: (a) Schematic illustration of the CNTFET biosensor fabrication process. (b) Gate voltage dependence of the normalized drain current $I_{d}$ for a CNTFET treated with Au NP-anti-HRP conjugates, BSA and HRP $\left(V_{d}=-0.01 \mathrm{~V}, \mathrm{HRP}\right.$ concentration $\left.=4.5 E-5 \mathrm{M}\right)$. (c) Drain-source voltage dependence of drain current $I_{d}$ for a CNTFET treated with HRP of concentrations ranging from $4.5 E-8 \mathrm{M}$ to $4.5 E-5 \mathrm{M}$.

Each antibody preferentially binds to a specific antigen, an interaction similar to that of a lock and key. The antigen (HRP) used in this study can only bind to the corresponding antibody (anti-HRP); therefore, if the structure of the antibody changes during the assembly process, there will be no binding events in the sensing process and no significant change in the drain current would have been observed. Moreover, the drain current change was observed to increase with the increasing HRP concentration. Based on the FET sensing results and the ELISA results, we conclude that the activity of antibodies on Au NPs is preserved during the electrospray and ESFDA assembly process.

\section{Conclusion}

The viability of antibodies on Au nanoparticles during an electrospray and electrostatic force directed assembly process is studied using ELISA and FET biosensing measurements. The activity of antibodies on Au nanoparticles is preserved in the assembly process as confirmed by the colored reaction in ELISA and the drain current change in FET measurements. Since the same assembly technique could successfully assemble Au nanoparticle-antibody conjugates onto carbon nanotubes and many other nanostructures in a controlled manner, the assembly method could be useful for protein labeling in various biosensor fabrication processes.

\section{Acknowledgments}

Financial support for this work was partially provided by the National Science Foundation (CMMI-0900509, CBET0803142, and ECCS-0708998). The SEM imaging was conducted at the Electron Microscope Laboratory of UWM. The e-beam lithography was performed at the Center for 
Nanoscale Materials of Argonne National Laboratory, which is supported by the US Department of Energy, Office of Science, Office of Basic Energy Sciences, under Contract no. DE-AC02-06CH11357. The authors thank Prof. D. P. Klemer for insightful discussion and helpful comments, Dr. H. A. Owen for technical support with SEM analyses, and Dr. L. E. Ocola for assistance in the electrode fabrication.

\section{References}

[1] R. J. Chen, S. Bangsaruntip, K. A. Drouvalakis, et al., "Noncovalent functionalization of carbon nanotubes for highly specific electronic biosensors," Proceedings of the National Academy of Sciences of the United States of America, vol. 100, no. 9, pp. 4984-4989, 2003.

[2] J. Li, H. T. Ng, A. Cassell, et al., "Carbon nanotube nanoelectrode array for ultrasensitive DNA detection," Nano Letters, vol. 3, no. 5, pp. 597-602, 2003.

[3] J. Wang, "Carbon-nanotube based electrochemical biosensors: a review," Electroanalysis, vol. 17, no. 1, pp. 7-14, 2005.

[4] M. Abe, K. Murata, A. Kojima, et al., "Quantitative detection of protein using a top-gate carbon nanotube field effect transistor," Journal of Physical Chemistry C, vol. 111, no. 24, pp. 8667-8670, 2007.

[5] B. L. Allen, P. D. Kichambare, and A. Star, "Carbon nanotube field-effect-transistor-based biosensors," Advanced Materials, vol. 19, no. 11, pp. 1439-1451, 2007.

[6] P. Avouris, "Molecular electronics with carbon nanotubes," Accounts of Chemical Research, vol. 35, no. 12, pp. 1026-1034, 2002.

[7] J. P. Kim, B. Y. Lee, S. Hong, and S. J. Sim, "Ultrasensitive carbon nanotube-based biosensors using antibody-binding fragments," Analytical Biochemistry, vol. 381, no. 2, pp. 193198, 2008.

[8] J. P. Kim, B. Y. Lee, J. Lee, S. Hong, and S. J. Sim, "Enhancement of sensitivity and specificity by surface modification of carbon nanotubes in diagnosis of prostate cancer based on carbon nanotube field effect transistors," Biosensors and Bioelectronics, vol. 24, no. 11, pp. 3372-3378, 2009.

[9] C. Li, M. Curreli, H. Lin, et al., "Complementary detection of prostate-specific antigen using $\operatorname{In}_{2} \mathrm{O}_{3}$ nanowires and carbon nanotubes," Journal of the American Chemical Society, vol. 127, no. 36, pp. 12484-12485, 2005.

[10] A. Star, J.-C. P. Gabriel, K. Bradley, and G. Gruner, "Electronic detection of specific protein binding using nanotube FET devices," Nano Letters, vol. 3, no. 4, pp. 459-463, 2003.

[11] M. T. Martinez, Y.-C. Tseng, N. Ormategui, I. Loinaz, R. Eritja, and J. Bokor, "Label-free DNA biosensors based on functionalized carbon nanotube field effect transistors," Nano Letters, vol. 9, no. 2, pp. 530-536, 2009.

[12] X. C. Dong, C. M. Lau, A. Lohani, et al., "Electrical detection of femtomolar DNA via gold-nanoparticle enhancement in carbon-nanotube-network field-effect transistors," Advanced Materials, vol. 20, no. 12, pp. 2389-2393, 2008.

[13] A. Star, E. Tu, J. Niemann, J.-C. P. Gabriel, C. S. Joiner, and C. Valcke, "Label-free detection of DNA hybridization using carbon nanotube network field-effect transistors," Proceedings of the National Academy of Sciences of the United States of America, vol. 103, no. 4, pp. 921-926, 2006.

[14] E.-L. Gui, L.-J. Li, P. S. Lee, et al., "Electrical detection of hybridization and threading intercalation of deoxyribonucleic acid using carbon nanotube network field-effect transistors," Applied Physics Letters, vol. 89, no. 23, pp. 232104-232106, 2006.

[15] J. H. Chen and G. H. Lu, "Controlled decoration of carbon nanotubes with nanoparticles," Nanotechnology, vol. 17, no. 12, pp. 2891-2894, 2006.

[16] G. H. Lu, L. Y. Zhu, P. X. Wang, et al., "Electrostatic-forcedirected assembly of Ag nanocrystals onto vertically aligned carbon nanotubes," Journal of Physical Chemistry C, vol. 111, no. 48, pp. 17919-17922, 2007.

[17] S. Mao, G. H. Lu, and J. H. Chen, "Coating carbon nanotubes with colloidal nanocrystals by combining an electrospray technique with directed assembly using an electrostatic field," Nanotechnology, vol. 19, no. 45, pp. 455610-455615, 2008.

[18] G. H. Lu, K. L. Huebner, L. E. Ocola, M. GajdardziskaJosifovska, and J. Chen, "Gas sensors based on tin oxide nanoparticles synthesized from a mini-arc plasma source," Journal of Nanomaterials, vol. 2006, Article ID 60828, 7 pages, 2006. 



The Scientific World Journal

Submit your manuscripts at

http://www.hindawi.com

\section{World Journal}

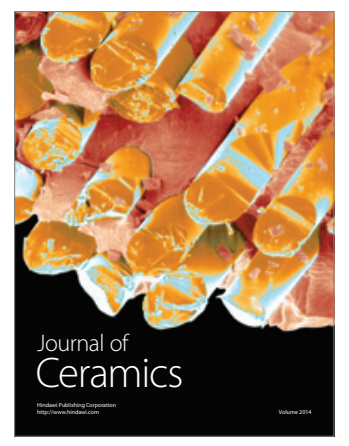

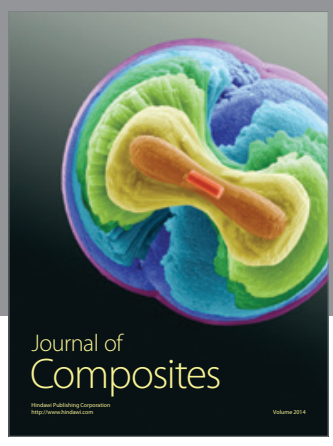
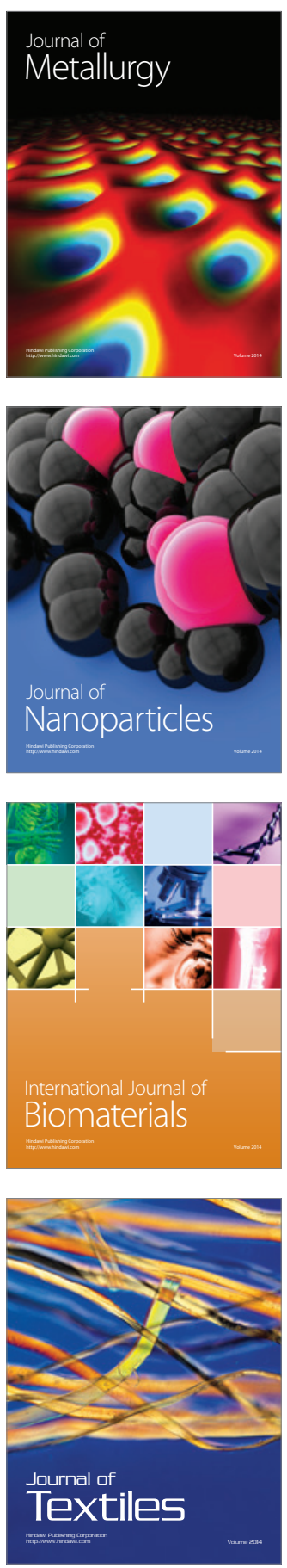